Fig. 1. Mean start, alley, and goal speed over the 5 trials preceding each drive shift and the 20 trials immediately following each shift. The numbers in the figure represent the hours of deprivation before and after each drive shift.

Newman-Keuls tests showed that the 22-h deprivation level was significantly faster than both the 12 - and 3-h deprivation levels ( $\mathrm{p}<.05$ : $r, 22)$, but that the 12- and 3-h levels did not differ significantly. For alley speed, both the $12-$ and $22-\mathrm{h}$ deprivation levels resulted in significantly faster running than the 3 -h deprivation level, $\mathrm{p}<.01(\mathrm{r}, 22)$ and $\mathrm{p}<.05(\mathrm{r}, 22)$, respectively. But the $12-$ and $22-h$ groups did not differ significantly.

Analysis of variance comparing the last 20 trials for the $12 \cdot, 22-$, and the final $3 \cdot h$ deprivation conditions showed a significant difference only for the alley measure, $F(2,22)=6.97, \quad p<.01$. Newman-Keuls tests showed that the overall $F$ was due to a significant difference between the 12and $3 \cdot \mathrm{h}$ deprivation levels ( $\mathrm{r}, 22), \mathrm{p}<.01$. Finally, a separate comparison of the two 3-h deprivation levels indicated that start speed at the second $3-\mathrm{h}$ level was significantly faster than that of the first $3-\mathrm{h}$ condition, $t(11)=2.38, p<.05$. For the other running measures there was no significant difference in response speeds at 3-h deprivation.

\section{DISCUSSION}

In general, performance changed rapidly following each shift in drive. This change was in evidence on the very first postshift trial, but the analysis was not performed on data involving fewer than five trials due to the relatively small $\mathrm{N}$ used in this study and the between-trials variability.

Terminal asymptotic performance was positively related to deprivation level when the first three deprivation levels were compared, but the relationship of performance to drive level was much weakened when the final 3 -h deprivation was compared with the 12- and 22-h levels. The fact that the second 3-h level immediately followed a 22-h deprivation level, where the highest performance level was attained, suggests that the drive-level/performance relationship is not a simple one and was affected by the sequence of exposing $\mathrm{Ss}$ following prolonged training.

Clearly these results show rapid shifts in performance with shifts in drive levels; however, the extent of this effect appears to decline with extensive training. Estes (1958, p. 52) argued that the amount of training is an important parameter of studies that compare performance at different drive levels. He argued that one would expect to find maximum differences

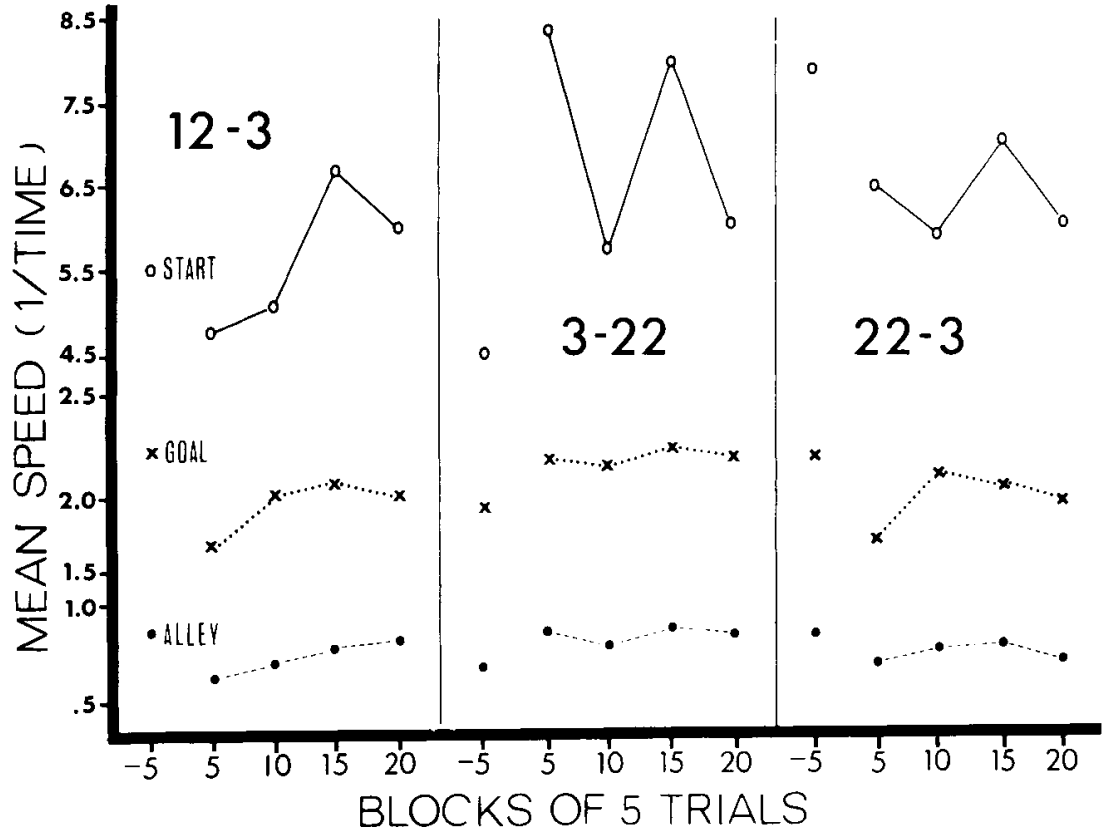

in performance at intermediate degrees of training, and smaller differences, or in some instances no difference, after very small or very large amounts of training.

In summary, these results indicated that performance change in the direction of drive shifts occur immediately following a change in deprivation level, when $\mathrm{Ss}$ are exposed to extensive training at each of several drive levels. However, these results are indeterminate as far as Estes's drive-stimulus theory is concerned due to the relatively small number of drive shifts, the limited range of these shifts, and the small number of Ss used. REFERENCES

COTTON, J. W. Running time as a function of amount of food deprivation. Journal of Experimental Psychology, 1953, 46, 188-198.
DEESE, J.. \& CARPENTER, J. A. Drive level and reinforcement. Journal of Experimental Psychology. 1951.42. 236-238.

ESTES. W. K. Stimulus-response theor: of drive In M. R. Jones (Ed.), Nebraska Simposium on Votilation. Lincoln: Lniversity of lebraskil Prew. 1958. Pp. 35-69.

HILLMAN, B.. HLNTER, W. S.. \& KIMBLF. G. A. The effect of drive level on the mazi performance of the white rat. Journal of Comparative \& Physiological Psychology. 1953.46. 87-89.

PORTER, J. J., MADISON, H. L., \& SENKOWSKI. P. C. Runway performance and competing responses as functions of drive level and method of drive measurement. Journal of Experimental Psychology, 1968, 78, 281-284. NOTE

1. Based upon the MS thesis of the first author and supported by NIMH Grant No. 17067-01 and grants from the University of Wisconsin-Milwaukee Graduate School.

\title{
Specific hungers in hyperphagic rats ${ }^{1}$
}

DOUGLAS G. MOOK, University of Virginia, Charlottesville, Va. 22901, and ELLIOTT M. BLASS, The Johns Hopkins University, Baltimore, Md. 21218

Preference-aversion functions for glucose, sucrose, and sodium chloride were determined using the single-stimulus, brief-exposure procedure. The functions generated by hyperphagic rats were similar to those generated by controls.

Specific hungers in the rat are characterized by highly reliable intake 
patterns (the "preference-aversion function"). This paper reports an investigation of preference-uversion drinking in rats made hyperphagic by ventromedial hypothalamic damage. We ask whether this striking disorder of regulatory behavior is accompanied by the abolition, reduction, or release of any of the identifiable factors that generate preference-aversion patterns in the normal animal (see Epstein, 1967, for review).

\section{SUBJECTS}

Eight adult female Sherman rats, weighing $230-290 \mathrm{~g}$ prior to operation, were housed and tested in individual cages. Ventromedial hypothalamic lesions were placed in four of them by passage of $1 \mathrm{~mA}$ $\mathrm{dc}$ for $30 \mathrm{sec}$ at the following flat-skull coordinates: $2 \mathrm{~mm}$ posterior to bregma, 0.5 to $0.7 \mathrm{~mm}$ lateral to the sagittal sinus, and 8.6 to $9.0 \mathrm{~mm}$ below the dural surface. Surgery was conducted under pentobarbital anesthesia.

\section{PROCEDURE}

Preference-aversion functions for glucose, sucrose, and sodium chloride were determined by the "single-stimulus" procedure of Weiner \& Stellar (1951). Each morning at 9 a.m., following overnight fluid deprivation, the animals were offered a single drinking fluid for $1 \mathrm{~h}$. Intake over that period was measured. Food was absent during the test period; and following it, food and fluid were withheld until 1 p.m. Water and food (Purina pellets) were then offered for a 4-h maintenance period. At 5 p.m., water (but not food) was removed and the rat deprived of fluid until the next morning's test. ${ }^{2}$ The various test solutions were presented in no fixed order.

After the completion of these observations, the operated rats were given ad lib access to food and water as a check on the effectiveness of the lesions. All gained weight rapidly, reaching final weight levels of from 652 to $727 \mathrm{~g}$. Therefore, the lesions were in all cases sufficient to produce vigorous hyperphagia and marked obesity.

\section{RESULTS}

Figure $\square$ shows the average intake of each test solution and of water by intact and hyperphagic rats. We see that for all three substances, the operated rats show the same intake pattern (the rising and falling preference-aversion function) as do normal rats. Their intakes of the solutions tend to be slightly higher than those of control rats, but their consumption of water is higher also; in fact, if the water-intake baselines were superimposed, the functions for normal and hyperphagic rats would be virtually identical. Putting it another way, we may say that the addition of these solutes to water has no differential effect on intake, as between normal and hyperphagic rats.

\section{DISCUSSION}

These data show that despite an insult to the brain sufficient to cause marked disturbance in other aspects of intake regulation, factors sufficient to produce normal preference-aversion drinking remain operative. This finding, in turn, implies that the hyperphagic rat remains responsive to both oral and postingestive controlling mechanisms, and that the two sets of factors interact in such animals as they do in neurologically intact ones.

Thus, the elevated intake of dilute sucrose solutions and the limited intake of concentrated saline solutions are both attributable primarily to taste factors: a positive influence in the one case, a negative one in the other (Mook, 1963). The integrity of these phenomena in the present case implies that taste factors continue to operate in the hyperphagic rat, as one would expect in view of the notorious "finickiness" that such rats display (Teitelbaum, 1955). On the other hand, the single-bottle saline "preference" results from the postingestive effects of dilute saline solutions, probably their

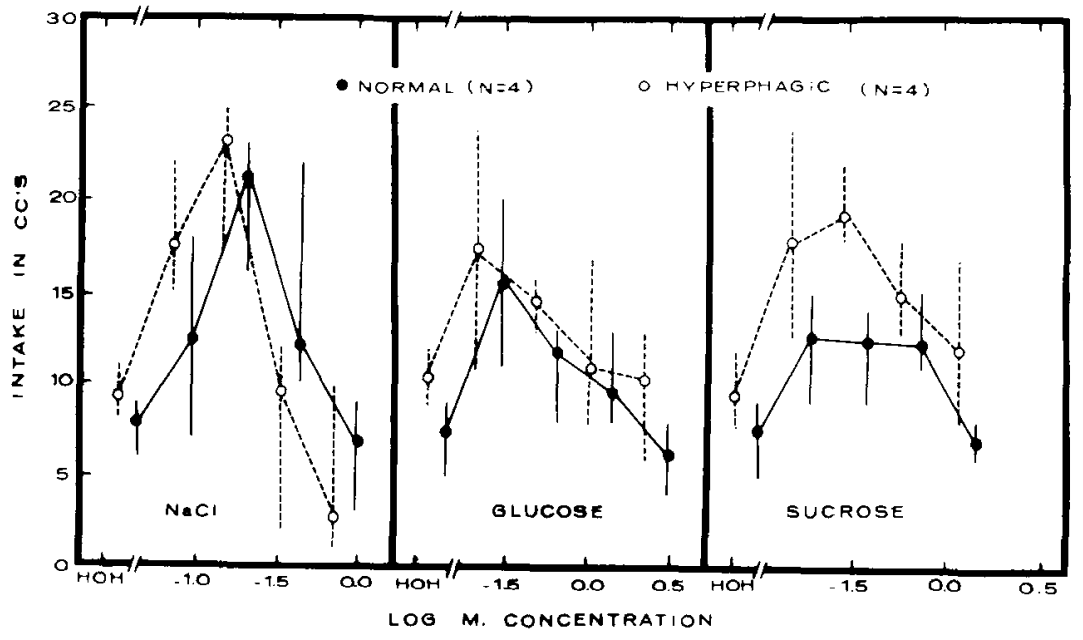

reduced effectiveness in slaking the rat's thirst (Mook, 1963). Therefore, hyperphagics remain sensitive to their hydrational states as implied in the findings of Corbit (1965).

Finally, a clear case of the interaction of oral and postingestive factors may be seen in the descending portions of the preference-aversion functions for the sugars. For example, rats drink relatively little of the more concentrated glucose solutions. This limited intake does not mean that such solutions are aversive as taste stimuli. For example, rats will select the higher of two glucose concentrations in a choice situation, even up to very high concentrations. This is true both of intact rats (Young \& Greene, 1953) and of hyperphagics (unpublished observations). Rather, the limited intake reflects the action of a postingestive mechanism that overrides a positive influence of taste (McCleary, 1953; Mook, 1963). And in the hyperphagic rats observed here, the intake of concentrated glucose solutions is just as sharply depressed as it is in intact animals, implying that, despite a presumed disorder in "satiety," postingestive satiety mechanisms remain potent in counteracting the stimulus to ingestion that a sweet taste provides.

\section{REFERENCES}

CORBIT, J. D. Hyperphagic hyperreactivity to adulteration of drinking water with quinine HCl. Journal of Comparative \& Physiological Psychology, 1965, 60,123-124.

EPSTEIN, A. N. Oropharyngeal factors in feeding and drinking. In C. F. Code (Ed.), Handbook of physiology. Sec. 6, Vol. I. Washington: American Physiological Society, 1967. Pp. 197-218.

MoCLEARY, R. A. Taste and postingestion factors in specific-hunger behavior.Journal of Comparative \& Physiological Psychology, $1953,46,411-421$.

MOOK, D. G. Oral and postingestional determinants of the intake of various solutions in rats with esophageal fistulas. Journal of Comparative \& Physiological Psychology, 1963, 56, 645-659.

TEITELBAUM, P. Sensory control of hypothalamic hyperphagia. Journal of Comparative \& Physiological Psychology, 1955, 48, 156-163.

WEINER, I. H., \& STELLAR, E. Salt preference in the rat determined by a single-stimulus method. Journal of Comparative \& Physiological Psychology. 1951, 44, 394-401.

YOUNG, P. T. \& GREENE, J. T. Quantity of food ingested as a measure of relative acceptability. Journal of Comparative \& Physiological Psychology, 1953, 46, 288-294. NOTES

1. Supported by PHS Grant No. MH 10766.

2. We have repeated the experiment adding overnight food deprivation to the fluid deprivation. The results are the same.

Fig. 1. Average intake and range of intakes of each test solution, for in tact and hyperphagic rats. For clarity, the control data are displaced slightly to the right. 